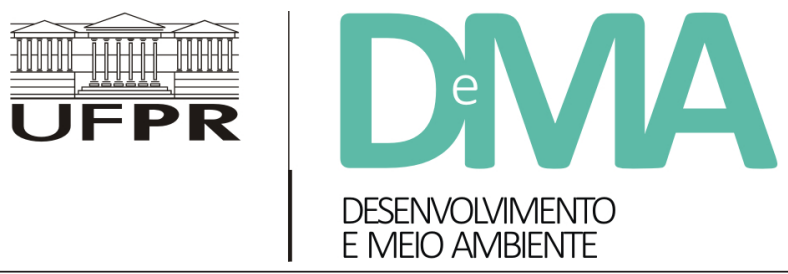

\title{
Análise da adequação do Plano Estadual de Gerenciamento Costeiro de Santa Catarina aos temas relevantes para a gestão costeira integrada
}

\section{Analysis of the Adequacy of the Santa Catarina Coastal State Plan of Management to the Relevant Topics for Integrated Coastal Management}

\author{
Victor GUIÃO ${ }^{1 *}$, Marinez Eymael Garcia SCHERER ${ }^{1}$ \\ ${ }^{1}$ Programa de Pós-Graduação em Geografia, Universidade Federal de Santa Catarina (UFSC), Florianópolis, SC, Brasil. \\ *E-mail de contato: victorguiao91@gmail.com
}

Artigo recebido em 31 de agosto de 2017, versão final aceita em 1 de novembro de 2017.

\begin{abstract}
RESUMO: Iniciativas que visam à melhoria da gestão da zona costeira são abundantes, tanto no Brasil como internacionalmente. Neste contexto, o Plano Estadual de Gerenciamento Costeiro de Santa Catarina (PEGC/SC) configura um importante instrumento para a gestão costeira catarinense, uma vez que propõe programas, linhas de atuação e planos de ação que visam organizar os usos e as atividades verificados na zona costeira do Estado. Entretanto, mesmo com o PEGC/SC, nota-se que a decadência da qualidade ambiental e os conflitos de uso e ocupação na costa de Santa Catarina persistem, sendo problemas frequentes: perda de biodiversidade, redução da área de habitat crítico, fragmentação de ecossistemas, redução da diversidade de paisagens naturais, erosão da linha de costa, dificuldade de acesso aos bens de uso público, entre outros. Por conta disso, a análise do Plano Estadual de Gerenciamento Costeiro de Santa Catarina (PEGC/SC) aparece como etapa necessária para que seja possível identificar as fragilidades deste instrumento de gestão e apontar alternativas aos problemas verificados. Este trabalho analisou o PEGC/SC a partir da verificação do atendimento de suas linhas de atuação em relação a temas considerados relevantes no âmbito de gestão costeira. Conclui-se que o PEGC/SC atende a maior parte dos temas relevantes para a gestão costeira, apesar de apresentar fragilidades que poderiam ser minimizadas pela real implementação das propostas já existentes no PEGC/SC, juntamente com a adição de outras três linhas de atuação ao plano, sendo elas: Incentivo à declaração clara de política pública para a gestão costeira catarinense; Estabelecimento de programas de capacitação continuada para gestores e técnicos costeiros; e Incentivo à produção e à organização de conhecimento e informação de interesse para a gestão costeira.
\end{abstract}

Palavras-chave: indicadores de gestão; gestão adaptativa; gestão costeira. 
ABSTRACT: Initiatives that aim to improve coastal zone management are abundant, both in Brazil and internationally. In this context, the State Plan for Coastal Management of Santa Catarina state (PEGC/SC, in Portuguese) is an important instrument for coastal management in Santa Catarina, since it proposes programs, goals and action plans, intending to organize the uses and activities observed in the state coastal zone. However, even with the structured PEGC/SC, it can be observed that the decline on environmental quality and conflicts of use and occupation still persist, being frequent problems: loss of biodiversity, reduction of critical habitat area, fragmentation of ecosystems, natural landscapes diversity reduction, coastline erosion, difficulty in accessing public areas, among other problems. Therefore, the analysis of the PEGC/SC appears as a necessary step to make it possible to identify the fragilities of this management tool and to point out alternatives to the verified problems. This work analyzed the PEGC/SC verifying the attendance of its goals in relation to topics considered relevant for the coastal management. It was concluded that PEGC/SC addresses most of the issues relevant to coastal management, despite its fragility. These weaknesses could be minimized by the real implementation of the existing PEGC/SC proposals. Alongside with the addition of three other goals to the plan, such as: Encouraging the clear declaration of public policy for coastal management in Santa Catarina; Establishment of continuing training programs for coastal managers and technicians; and Incentive to the production and organization of knowledge and information of interest for coastal management.

Keywords: management indicators; adaptive management; coastal management.

\section{Introdução}

No âmbito da Política Nacional do Meio Ambiente (PNMA), estabelecida pela Lei Federal $n^{\circ}$ $6.938 / 81$, a zona costeira brasileira aparece como área fundamental para a conservação de recursos vivos, sociais, culturais e paisagísticos (Brasil, 1981). Além disso, este ambiente configura parcela importante do território nacional por fatores como a fragilidade de seus ecossistemas, a grande concentração de população e o intenso desenvolvimento econômico (Moraes, 1999; Freire, 2002; SPG, 2010a). Tendo em vista esta importância, a constituição federal brasileira classifica a zona costeira como "patrimônio nacional" e garante atenção especial para assuntos relacionados a aspectos físico-naturais, socioeconômicos e político-administrativos deste ambiente.

Neste sentido, o Plano Nacional de Gerenciamento Costeiro, atualmente em sua segunda versão (PNGC II), é operacionalizado no âmbito do Programa Nacional de Gerenciamento Costeiro (GERCO) e estabelece normas gerais que visam ao ordenamento do uso dos recursos naturais e da ocupação dos espaços costeiros (Asmus et al., 2006). Para isso, o PNGC considera instrumentos de gestão que permitem a efetivação das ações relacionadas à costa, sendo um deles os Planos Estaduais de Gerenciamento Costeiro (PEGC). Entretanto, como assinalam Andrade e Scherer (2014), embora o GERCO esteja em implantação desde 1987 e conte com uma base legal fundamentada na Lei Nacional de Gerenciamento Costeiro ( $\mathrm{n}^{\circ} 7.661 / 88$ ), as ações de operacionalização do programa são ainda bastante incipientes em Santa Catarina e no país. As autoras ressaltam, também, que a não execução das ações propostas pelo GERCO tem facilitado práticas não sustentáveis, que intensificam a perda de recursos naturais e serviços ambientais costeiros.

De acordo com o PNGC II, todos os instrumentos brasileiros de gestão costeira devem ser replicados nos estados e municípios. Apesar das dificuldades verificadas nos processos de aplicação 
destes instrumentos pelos estados costeiros (Andrade \& Scherer, 2014), o Estado de Santa Catarina iniciou, no ano de 2010, a implementação do Plano Estadual de Gerenciamento Costeiro catarinense (PEGC/SC) e desenvolveu o Plano de Gestão da Zona Costeira (PGZC/SC). Por intermédio deste documento - mais especificamente do Plano de Gestão Regional (PGR/SC), que engloba todos os setores do litoral catarinense -, o PEGC/SC propõe programas, linhas de atuação e planos de ação por meio dos quais os objetivos da gestão costeira estadual podem ser alcançados. Esta estruturação encontra-se exemplificada na Figura 1. ção" (SPG, 2010b, p. 8). Contudo, Santa Catarina aparece como um Estado em que o panorama de degradação ambiental costeira é preocupante, tendo em vista a intensa ocupação do litoral, a aceleração dos efeitos das mudanças climáticas sobre a zona costeira, o aumento da atividade turística e o não aproveitamento dos instrumentos de gerenciamento costeiro (Pollete \& Cavedon, 2001; Andrade \& Scherer, 2014).

Como reflexo destes fatos, é comum verificar na zona costeira catarinense problemas como a perda de biodiversidade, a redução da área de habitat crítico, a fragmentação de ecossistemas, a redução

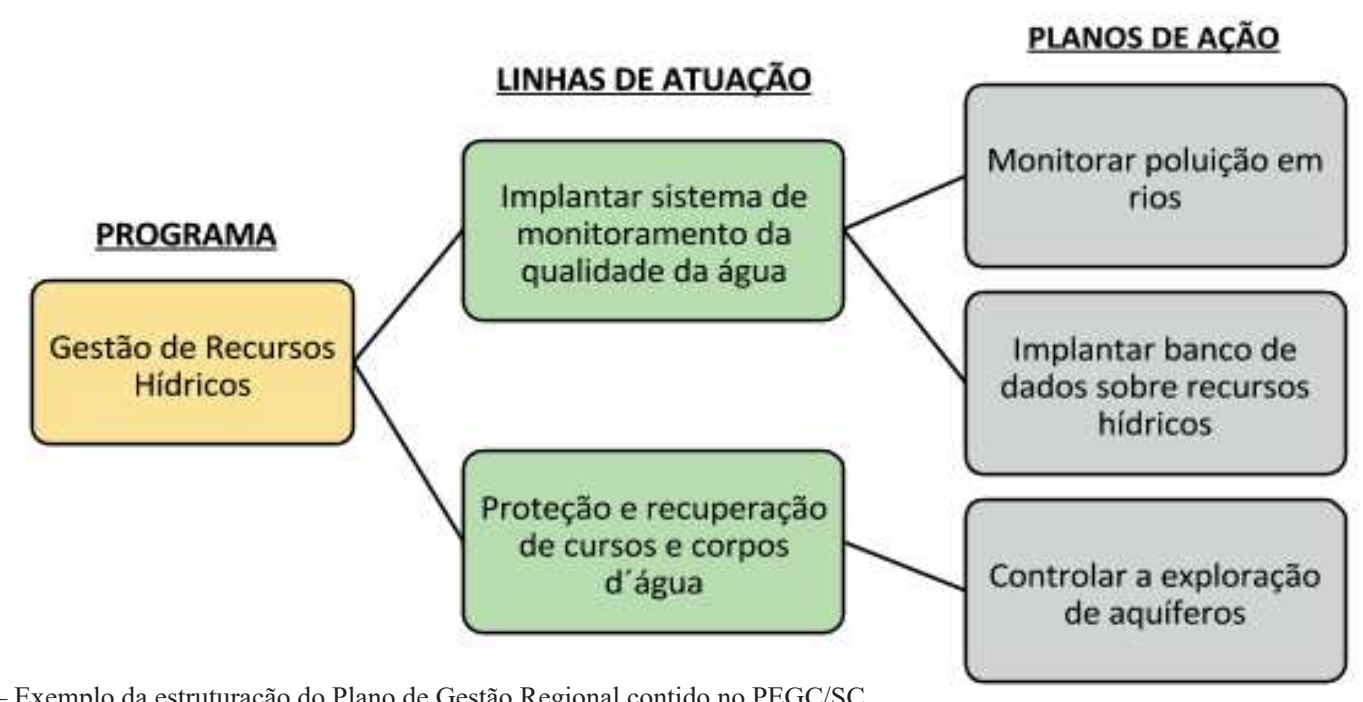

FIGURA 1 - Exemplo da estruturação do Plano de Gestão Regional contido no PEGC/SC. FONTE: elaboração dos autores, 2017.

Nesse sentido, PEGC/SC apresenta a totalidade de 13 programas, 40 linhas de atuação e 85 planos de ação, por meio dos quais objetiva "ordenar as diferentes atividades e usos do território costeiro a fim de promover o desenvolvimento socioeconômico sustentável e que garanta a conservação dos recursos naturais e a qualidade de vida da popula- da diversidade de paisagens naturais, a erosão da linha de costa e a dificuldade de acesso aos bens de uso público (Pollete \& Cavedon, 2001; Scherer, 2013). Este cenário reforça, portanto, a necessidade de se analisar as propostas existentes no PEGC/SC para que seja possível identificar as fragilidades da gestão costeira catarinense e apontar novas alterna 
tivas aos problemas verificados.

Assim, o objetivo principal deste trabalho é analisar o PEGC/SC a partir da verificação do atendimento de suas linhas de atuação em relação a temas considerados relevantes no âmbito da gestão costeira destacados pela literatura especializada. Com este esforço, almeja-se contribuir com diretrizes para a melhoria da gestão costeira no Estado catarinense.

\section{Metodologia}

Foi realizado um levantamento de dados baseado no documento oficial disponibilizado pelo governo de Santa Catarina (SPG, 2010b), a fim de identificar as linhas de atuação propostas.

A fim de analisar se as propostas do PEGC/ $\mathrm{SC}$ atendem a temas relevantes para a gestão costeira, foram analisadas as publicações de Barragán (2004) e Olsen et al. (1999). Estes autores foram selecionados para a análise pois apresentam tópicos semelhantes que, caso devidamente atendidos, contribuiriam para o sucesso de processos de gestão costeira, uma vez que "propõem que existem princípios básicos no processo e estratégias de gestão integrada da zona costeira que devem estar presentes nas estratégias de gestão e políticas públicas" (Andrade \& Scherer, 2014, p. 148). No entanto, é importante ressaltar que os temas apontados pelos autores analisados são eminentemente estratégicos. Assim, temas operativos não foram avaliados.

Este trabalho utilizou como referência os temas descritos por Barragán (2004), os quais correspondem ao Decálogo da Gestão Costeira (Figura 2). Além destes, também foram considerados como referência os temas relevantes apontados por Olsen et al. (1999) (Figura 3), os quais correspondem a assuntos que devem ser avaliados, de acordo com agências de fomento e organizações internacionais, a fim de estabelecer o grau de maturidade da gestão costeira. Como dito anteriormente, estes temas apresentam grande semelhança com aqueles propostos por Barragán (2004).

A análise do PEGC/SC foi realizada a partir da elaboração do Quadro de Fragilidades, em que é exposto o atendimento dos temas considerados relevantes pelos autores analisados por parte do PEGC/ SC e suas linhas de atuação. A análise do Quadro de Fragilidades levou em consideração a presença/ ausência de linhas de atuação do PEGC/SC que se relacionem com os temas considerados relevantes. É importante ressaltar que, devido ao número de temas propostos por cada um dos autores ser diferente (10 para Barragán e 16 de Olsen), os mesmos foram agrupados no Quadro de Fragilidades por semelhança. Por sua vez, as linhas de atuação do $\mathrm{PEGC/SC} \mathrm{foram} \mathrm{representadas} \mathrm{por} \mathrm{números.}$

Deve-se ressaltar que as 40 linhas de atuação do PEGC/SC são apresentadas no Quadro de Fragilidades de maneira resumida. Adotou-se duas ou três palavras-chave da linha de atuação para identificar cada uma delas. Alguns exemplos são: "Fiscalização e Monitoramento dos Recursos Naturais", que está identificada como "Fiscalização Recursos"; "Proteção e Recuperação de Recursos e Corpos d'Água", que está identificada como "Proteção Água"; "Incentivo à Recuperação de Áreas Degradadas", que está identificada como "Incentivo Recuperação".

A partir das fragilidades identificadas, este trabalho sugeriu novas linhas de atuação que buscam suprir, caso consideradas no PEGC/SC, as necessidades do processo de gestão costeira vigente em Santa Catarina. 

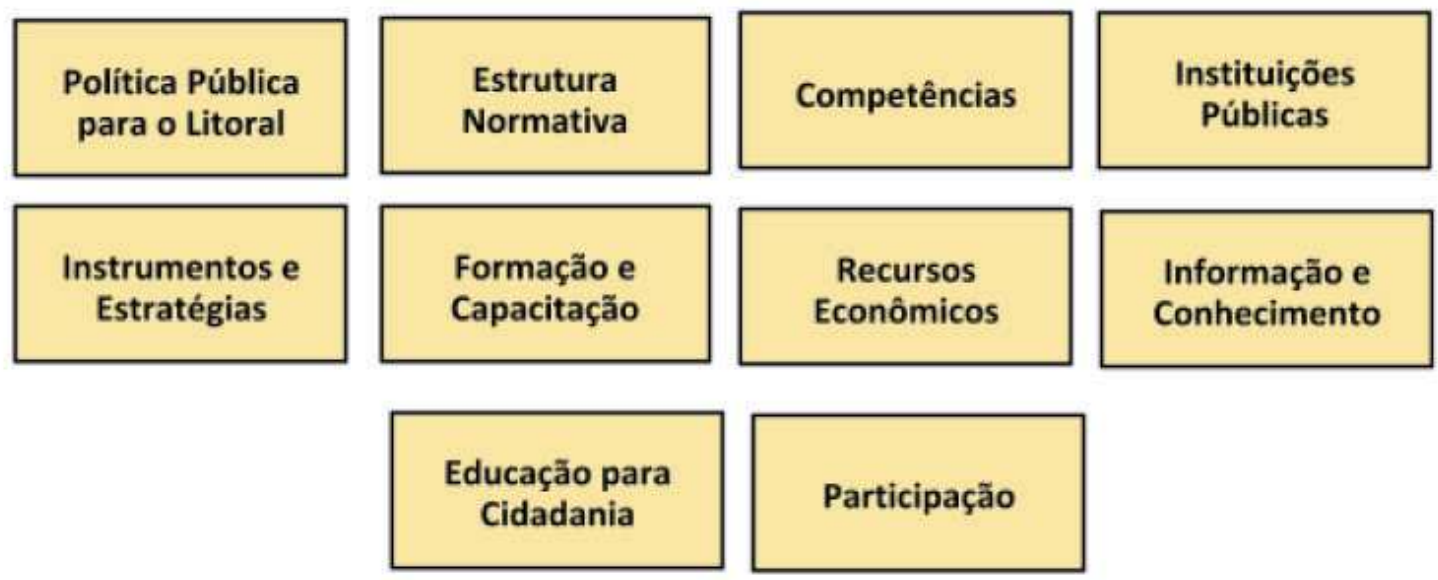

FIGURA 2 - Temas relevantes para a gestão costeira segundo Barragán (2004). FONTE: Barragán (2004)

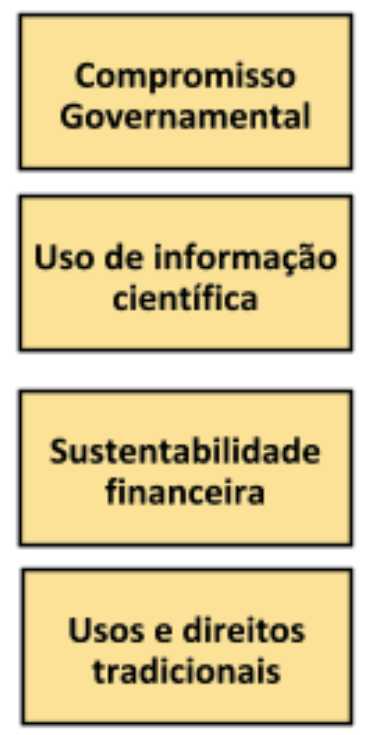

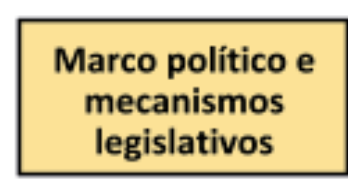
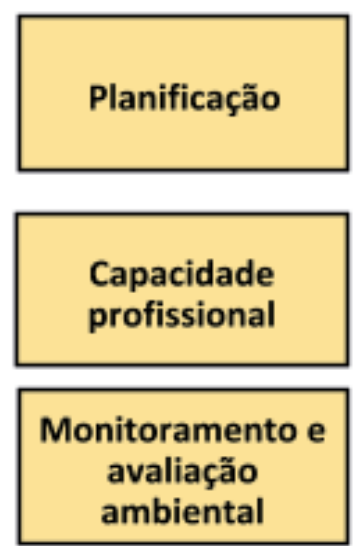

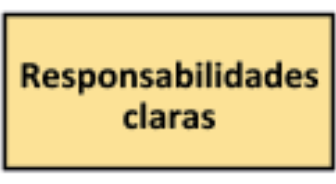

Tomada de decisões e gestão participativa

\section{Educação pública e conscientização}

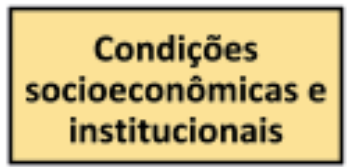

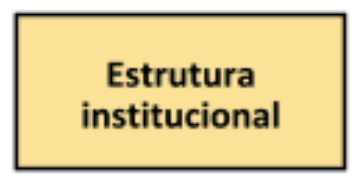

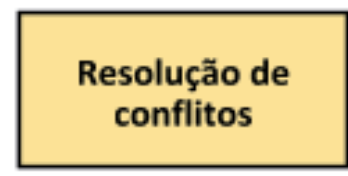

Divulgação pública

Transferência de
conhecimento/
experiência

FIGURA 3 - Temas relevantes para a gestão costeira segundo Olsen et al. (1999).

FONTE: Olsen et al. (1999)

\section{Resultados e discussão}

A análise do PEGC/SC indica que, de forma geral, o processo de gestão costeira estabelecido em
Santa Catarina apresenta grande compatibilidade com os temas considerados relevantes por Barragán (2004) e Olsen et al. (1999). Entretanto, nota-se que o plano catarinense não possui linhas de atuação suficientes para que todos os temas propostos pelos 
autores sejam atendidos.

Como indica o Quadro de Fragilidades (Tabela 1), a maior parte dos temas relevantes - total de 10 entre os 13 temas analisados após o agrupamento por semelhança - é atendida pelo PEGC/SC, com a existência de uma ou mais linhas de atuação que abordam assuntos diretamente relacionados àqueles sugeridos pelos autores. Como exemplo, pode-se citar o tema "Instrumentos e Estratégias", de Barragán (2004), denominado por Olsen et al. (1999) como "Planificação". Este tema foi considerado atendido pelo $\mathrm{PEGC/SC}$ por conta da existência das linhas de atuação "Incentivo à adoção de planos e projetos integrados na zona costeira", "Promoção

TABELA 1 - Quadro de Fragilidades, que mostra o atendimento aos temas relevantes para a gestão costeira por parte das propostas do PEGC/SC.

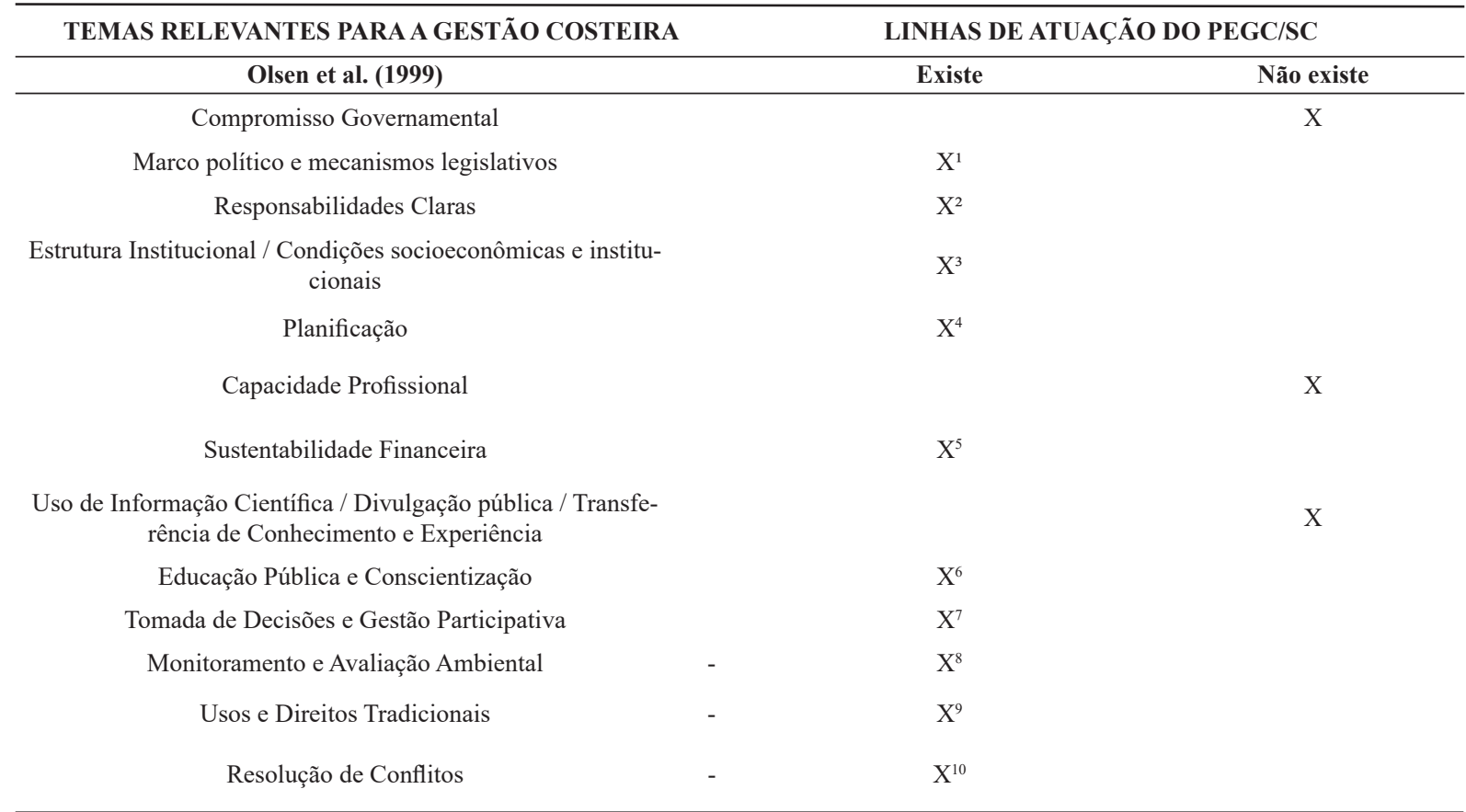

Legenda (Linhas de Atuação que atendem aos temas relevantes):

$\mathrm{X}^{1}$ - Revisão Legal;

$\mathrm{X}^{2}$ - Equipe;

$\mathrm{X}^{3}$ - Equipe, Recurso Financeiro; Planos Integrados;

$\mathrm{X}^{4}$ - Planos Integrados; Promoção Crédito. Integração Bacias;

Articulação Planos;

$\mathrm{X}^{5}$ - Recurso Financeiro;

$\mathrm{X}^{6}$ - Educação Ambiental;

$\mathrm{X}^{7}$ - Comunicação; Participação Pública; Integração;
$\mathrm{X}^{8}$ - Definição Espécies; Fiscalização; Recursos; Controle Espécies; Sistemas G.A.; Gestão UCs; Incentivo; Recuperação; Monitoramento Água; Fiscalização Pesca;

$\mathrm{X}^{9}$ - Quilombolas, Conservação Histórico; Participação Comunidades;

$\mathrm{X}^{10}$ - Compatibilização; Controle; Contaminantes; Monitoramento Socioeconômico.

FONTE: elaboração dos autores, 2017 
de mecanismos de crédito para as linhas de atuação dos programas de caráter público ou privado", "Integração do gerenciamento costeiro com a gestão de bacias hidrográficas" e "Articulação do Plano Estadual de Gerenciamento Costeiro com os Planos Municipais de Gerenciamento de Risco".

Contudo, não foi constatada a existência linhas de atuação no $\mathrm{PEGC/SC}$ que se relacionem com três dos 13 temas relevantes, sendo eles: "Política Pública para o Litoral", "Formação e Capacitação" e "Informação e Conhecimento" (Barragán, 2004), respectivamente considerados por Olsen et al. (1999) como "Compromisso Governamental", "Capacidade Profissional" e "Uso de Informação Científica/Divulgação Pública/Transferência de Conhecimento e Experiência". Nestes casos, a ausência de linhas de atuação revela fragilidades que podem ser determinantes para a existência dos problemas constantemente verificados na zona costeira de Santa Catarina.

É interessante ressaltar que as fragilidades evidenciadas na Tabela 1 foram identificadas com base em critérios eminentemente estratégicos, propostos pelos autores, e que se relacionam com a natureza estruturante da gestão costeira e visam ao incentivo à adoção de políticas claras, normativa pertinente e formação e capacitação de agentes públicos, por exemplo (Scherer et al., 2014). Sendo assim, temas de caráter operativo não foram avaliados, apesar de grande parte das linhas de atuação existentes no PEGC/SC possuir objetivos de caráter operativo, como, por exemplo, a implantação de sistemas de saneamento e a melhoria dos sistemas de transporte. Com base nos autores de referência, considera-se que ações operativas são importantes para a manutenção da qualidade ambiental, mas não são estruturantes da gestão costeira.
Ao não possuir uma política pública explícita, clara e divulgada, sendo de conhecimento de toda a comunidade costeira catarinense, a gestão costeira se torna frágil frente à distribuição de recursos públicos, mudanças na estrutura do governo e falta de equipe técnica, por exemplo. Estes problemas já ocorrem e foram destacados por Andrade \& Scherer (2014).

Da mesma forma, Andrade \& Scherer (2014) também apontaram a necessidade de formação de pessoal capacitado e a falta de equipe técnica para a gestão da zona costeira em Santa Catarina. Barragán (2016) ressalta que a lacuna existente na formação e na capacitação de gestores talvez seja um dos problemas mais importantes da gestão costeira na região latino-americana. Deste modo, de acordo com as considerações dos autores consultados, Santa Catarina poderia aprimorar a formação dos gestores por meio de ações contidas em um Plano de Gestão.

Por sua vez, a falta de uma linha de atuação que demande um maior conhecimento técnico-científico do litoral, assim como a divulgação deste conhecimento, também fragiliza o PEGC/SC, uma vez que a base da gestão costeira depende de informação e conhecimento específico (Barragán, 2004). O próprio diagnóstico da zona costeira de Santa Catarina, disponibilizado pela Secretaria de Planejamento e Gestão do Estado de Santa Catarina (SPG, 2010a), aponta para lacunas de informação importantes na porção marinha do litoral. Assim, criar uma linha de atuação no PEGC/SC que promova a obtenção destas informações é de grande relevância para a gestão.

Desta maneira, tendo em vista as fragilidades da gestão costeira catarinense - constatadas a partir da inexistência de linhas de atuação suficientes para 
atender a totalidade dos temas relevantes sugeridos por Barragán (2004) e Olsen et al. (1999), a sugestão de linhas de atuação ao PEGC/SC configura uma importante tentativa de gerar diretrizes que possam contribuir com a gestão costeira integrada em Santa Catarina.

\section{Considerações finais}

A análise das linhas de atuação propostas indica que o PEGC/SC direciona as atenções do processo de gestão costeira catarinense para temas considerados importantes por Olsen et al. (1999) e Barragán (2004), ainda que não de maneira abrangente. Entretanto, apesar da compatibilidade entre as linhas de atuação do $\mathrm{PEGC/SC}$ e grande parte dos temas propostos pelos autores, é possível perceber fragilidades no processo de gestão costeira catarinense.

Tais fragilidades se baseiam na falta de atendimento a determinados temas de Olsen et al. (1999) e Barragán (2004), de caráter eminentemente estratégico, e podem ser sanadas com a adição de novas linhas de atuação no PEGC/SC. Sendo assim, com base na análise da Tabela 1, este trabalho sugere as seguintes linhas de atuação: "Incentivo à declaração clara de uma política pública para a gestão da zona costeira catarinense"; "Estabelecimento de programas de capacitação continuada para técnicos e gestores costeiros"; "Incentivo à produção e à organização de conhecimento e informação de interesse para a gestão costeira". No entanto, estas linhas de atuação adicionais, bem como as demais já constantes no Plano, devem ser, de fato, implementadas. A partir da real implementação das ações estipuladas, a gestão da zona costeira em Santa
Catarina poderá contribuir para o desenvolvimento sustentável.

\section{Referências}

Andrade, J.; Scherer, M. E. G. Decálogo da gestão costeira para Santa Catarina: avaliando a estrutura estadual para o desenvolvimento do Programa Estadual de Gerenciamento Costeiro. Desenvolvimento e Meio Ambiente, 29, 139-154, 2014.

Asmus, M. L.; Kitzmann, D.; Laydner, C.; Tagliani, C. R. Gestão Costeira no Brasil: instrumentos, fragilidades e potencialidades. Gestão Costeira Integrada, 5, 52-57, 2006. Disponível em: <http://repositorio.furg.br/bitstream/ handle/1/2053>.

Barragán, J. M. M. Las áreas litorales de España: del análisis geográfico a la gestión integrada. Barcelona: Editorial Ariel S.A., 2004.

Barragán, J. M. M. Política, gestão e litoral: uma nova visão da gestão integrada de áreas litorais. Madrid: Editorial Tébar Flores, 2016. $685 \mathrm{p}$.

Brasil. Lei $n^{\circ} 6.938$, de 1981. Dispõe sobre a Política Nacional do Meio Ambiente e dá outras providências. Brasília: D.O.U de 16/05/1981.

Freire, O. D. da S. Projeto Orla: fundamentos para gestão integrada. Brasília: MP/SPU, 2002.

Moraes, A. C. R. Contribuições para a gestão da zona costeira do Brasil: elementos para uma Geografia do Litoral Brasileiro. São Paulo: Hucitec/Edusp, 1999.

Olsen, S.; Lowry, K.; Tobey, J. A manual for assessing progress in coastal management. Coastal Management Report, 2211, 1999.

Pollete, M.; Cavedon, F. Construindo o Desenvolvimento Sustentável para o Municipio de Bombinhas. Centro de Ciências Tecnológicas da Terra e do Mar-UNIVALI. Itajaí: CGMA, 2001. 115p.

Scherer, M. Gestão de Praias no Brasil: Subsídios para uma Reflexão. Gestão Costeira Integrada, Florianópolis, 13., 
3-13, 2013. Disponível em: <http://www.aprh.pt/rgci/pdf/ rgci-358_Scherer.pdf $>$.

Scherer, M.; Andrade, J.; Emerim, E. G.; Felix, A.; Oliveira, T. C. R.; Mondl, H. B.; Lima, F. A. V. Prioritizing actions for coastal management: A methodological proposal. Ocean \& Coastal Management, 91, 17-22, 2014. doi: 10.1016/j. ocecoaman.2014.01.012
SPG - Secretaria de Planejamento e Gestão do Estado de Santa Catarina. Implantação do Plano Estadual de Gerenciamento Costeiro: Diagnóstico Socioambiental, 2010a. Disponível em: $<$ http://www.spg.sc.gov.br/gerco/diagnostico-socio-ambiental>. Acesso em: out. 2017.

SPG - Secretaria de Planejamento e Gestão do Estado de Santa Catarina. Implantação do Plano Estadual de Gerenciamento Costeiro: Plano de Gestão Regional, $2010 \mathrm{~b}$. Disponível em: <http://www.spg.sc.gov.br/visualizar-biblioteca/acoes/gerco/atualizacao-de-outubro-de-2013/ 31-plano-gestao-regional-fase-i/file>. Acesso em: jun. 2017. 